VEHICLE REIDENTIFICATION IN A CONNECTED VEHICLE ENVIRONMENT USING MACHINE LEARNING ALGORITHMS

\title{
Zuoyu Miao
}

University of Arizona

System and Industrial Engineering Department

1127 E. James E. Rogers Way, Tucson, AZ 85721, USA

Email: zymiao@email.arizona.edu

\section{K. Larry Head}

Professor

University of Arizona

System and Industrial Engineering Department

1127 E. James E. Rogers Way, Tucson, AZ 85721, USA

Email:klhead@email.arizona.edu

\section{Byungho Beak}

University of Arizona

System and Industrial Engineering Department

1127 E. James E. Rogers Way, Tucson, AZ 85721, USA

Email: beak@email.arizona.edu

Word count: 6000 words +6 figures/tables x 250 words $=7500$ words

Submission Date: 2-24-2018 


\begin{abstract}
Deployment of Connected Vehicles will become available for most American cities in next 10 to 20 years. The applications (e.g. mobility, safety, environment) are constantly receiving vehicle data. The current ID protection mechanism assumes a vehicle's ID changes every 5 minutes, the topic of re-matching vehicles is of interests in privacy protection and performance measure research. This paper explores the possibility of re-matching connected vehicles' ID using popular machine learning techniques, including Logistic Regression (LR), Linear Discriminant Analysis (LDA), Quadratic Discriminant Analysis (QDA), Linear and Nonlinear Support Vector Machine (SVM) and Nearest Neighbor algorithms. An experiment is conducted using a microscopic traffic simulation model through a Software-In-the-Loop technique. The best average mis-matching rate is $14 \%$. To assess potential factors' effects on matching accuracy, a Poisson mixed regression model is analyzed under the Bayesian inference framework. Findings are: different matching algorithms vary in matching performance and the Linear SVM, the QDA and the LDA have the best accuracy results; traffic volume and market penetration rate have little impact on matching results; location and number of vehicles to be matched are considered significant. The results make the performance measure of future CV applications feasible and also suggest that more secure mechanisms are in need to protect the public.
\end{abstract}

Keywords: Connected Vehicle, ID Matching, Privacy Protection, Machine Learning, Bayesian Inference, Poisson Mixed Regression 


\section{INTRODUCTION}

Connected Vehicle (CV) technology has made the realization of Vehicle to Vehicle (V2V) and Vehicle to Infrastructure (V2I) communication possible. New applications are emerging under the V2I framework that enable the Road Side Unit (RSU) to collect real-time vehicle data to improve traffic mobility, as well as safety. According to the FHWA Vehicle-to-Infrastructure Deployment Guidance (1), limited deployment of RSUs and equipped vehicles has begun and is expected to become widespread over the next 10 to 20 years. With the rapid development of $\mathrm{CV}$ technologies, more and more users' data will be collected.

While the enriched data facilitates CV applications, publicly publishing data could potentially pose significant privacy and security concerns. The Federal Trade Commission (FTC) and the NHTSA held an open workshop on June 28, 2017 in Washington, D.C to discuss such privacy and security issues (2). The spatial and temporal attributes of trajectories can be used as powerful quasi-identifiers linking personal identification information (PII). Movements of connected vehicles can be observed by chance or on purpose, even with few observations. By matching trajectories having the same spatial and temporal attributes, a user' trip information may be inferred, including home and work addresses. Therefore, third party's access to such trajectory data may cause serious privacy and security threats. In the connected vehicle environment, the equipped vehicles generate random identities every 300 seconds to break one's trajectory into pieces and mix with other vehicles. However, even with such a pseudonym protecting mechanism, exposure of the de-identified vehicle trajectory data to adversaries is still considered to be risky. This paper serves as an evidence to show that strict $\mathrm{CV}$ protection strategies are essential to secure consumer privacy.

When new ITS applications are implemented in the CV environment, the individual connected vehicle's travel time and delay are important measures for performance analysis. Without a vehicle ID matching process, the real-world system-level performance measures cannot be fully implemented, since one vehicle may have different IDs as it moves among intersections. Therefore, matching connected vehicles'IDs becomes desirable. More research work is needed in vehicle ID matching in the quickly maturing $\mathrm{CV}$ environment as the newly emerged technologies (V2I and V2V communications) are scheduled to be deployed nationwide in the next few years.

This paper explores trajectory matching possibilities by implementing several machine learning classification techniques and systematically assessing factors potentially affecting the matching accuracy. The organization of this paper is: The Literature Review provides a review on vehicle matching and experiment analysis of random factors; the Description of Connected Vehicle Reidentification and Machine Learning Methods introduce algorithms for CV matching; the Results consists of model specification, validation and factor effect analysis; the Conclusion provides a summary and future work.

\section{LITERATURE REVIEW}

To the best of our knowledge, there is no literature on vehicle reidentification using CV data. Significant research has been focused on maintaining the vehicle ID as it travels through multiple video camera sites. Zeng and Crisman (3) proposed to match vehicles using body colors and the matching accuracy was $16.42 \%$ with an 8 -vehicle test sample. Kogut and Trivedi (4) investigated color features and the spatial organization of vehicle platoons together for vehicle matching. They 
achieved an accuracy rate of $45 \%$. But their research work was based on a small number of samples and did not consider the site location impact on matching accuracy. Later, additional vehicle features were considered (5), such as external dimensions, points of optical demarcation, etc. Another approach was based on a sparse representation algorithm which was originally proposed as a facial recognition method (6). The authors claimed an accuracy of $57.84 \%$. The limitations of video-based vehicle matching methods include illumination variations, occlusion phenomena and object overlapping. Such difficulties, and the cost of video detector deployment and maintenance, make this vehicle matching method unfavorable for transportation engineering practitioners. Some research has used unique vehicle IDs for identification. Examples include matching unique Bluetooth MAC addresses (7-13), GPS device addresses (14) and magnetic signatures (15-18). However, not all these ID matching facilities are widely deployed in the U.S.. Another approach is vehicle trajectory tracking. The general routine is to detect vehicles in a picture by feature classification and then to track the object using Kalman filtering and its variants. A thorough literature review was conducted in on-road vision-based vehicle detection, tracking, and behavior understanding by Sivaraman, et. al. (19). Their review found that the tracked object is always confined in one camera's view angle and only suitable for continuous vehicle trajectory estimation. In the $\mathrm{CV}$ environment, vehicle features are automatically and precisely recorded in Basic Safety Messages (BSMs) and extracting them is effortless compared to image processing. This advantage of structured data provides the possibility of matching vehicle ID in a more complex real-life environment with better accuracy comparing to the unstructured image data.

To systematically assess the performance of matching methods and other factors, it is helpful to use designed experiments. The traditional experiment analysis is the Analysis of Variance (ANOVA) approach. Even though this classical framework has served the simulation and field data analytics for decades, it also has two limitations. First, this approach requires well balanced sample sizes with the number of observations for each set of conditions being almost equal. In a real data collection process, observations are often missing due to human error, database malfunction, and condition limitations. Some modifications to the ANOVA were proposed by using approximate methods $(20 ; 21)$, but they are only appropriate for situations where the dataset is mildly unbalanced (22). Second, non-normal data is usually transformed to make it appear normal (23). The transformation should be conducted very carefully, because it can fail if count data contains many zero values, model assumptions are violated, or the scope of inference is limited. To account for these disadvantages, the Residual Maximum Likelihood (REML) method is often employed for parameter estimation as an alternative to the Maximum Likelihood (ML) method when both random and fixed factors are considered in the General Linear Mixed Model $($ GLMM) (24). Since the likelihood equations are in highly non-linear, closed analytical solutions are not available. Approximation methods include the Quasi-Likelihood (QL) approach which uses a Taylor series expansion (25-27) and the Gauss-Hermite Quadrature (GHQ) method which uses optimal subdivisions to numerically evaluate the integrand (28). The QL approach induces bias for inference purposes and the GHQ approaches work poorly with more than two random factors (29). Instead of trying to solving the likelihood functions, the Markov Chain Monte Carlo (MCMC) algorithm (30) generates random samples from the distributions of parameter values of factor effects and is free of these drawbacks.

In this paper, with the help of Software-In-the-Loop (SIL) simulation, CV trajectory data is collected through a VISSIM simulation. Seven supervised machine learning classification algorithms are applied with considerations of different scenario settings. To explore the factors 
affecting the matching results, a designed experiment and its corresponding statistical analysis are presented.

\section{DESCRIPTION OF CONNECTED VEHICLE REIDENTIFICATION}

Typically, RSUs are mounted on top of traffic light signal pole of equipped intersections. Using Dedicated Short Range Communication (DSRC), approaching vehicles send BSMs up to 10 times per second to an RSU. According to the Multi-Modal Intelligent Traffic Signal System (MMITTS) project (31), for purposes of traffic signal control and performance measures, BSMs are collected and stored every second when vehicles are in the range of DSRC which is between 300 and 600 meters. More details of this database design can be found on Page 61 of the final report (31).

According to the Society of Automotive Engineers (SAE) J2735 standard (32), the BSM data contains vehicle position, time, speed, heading acceleration, brake system status, phases, etc. In this research, only the vehicle position and the timestamp are used to match CV IDs and other vehicle properties are not included in the matching algorithms. This is due to two considerations: First, vehicle physical properties can serve as the quasi-ID to match vehicles before the machine learning techniques are applied. This pre-process can reduce the number of vehicle to be matched. In the situation where each vehicle has its unique properties, the reidentification can be done without further matching algorithms. But the methods proposed in this paper can deal with more complex scenarios in which many vehicles share common physical properties. Second, incorporating the physical properties as covariates will cause computation difficulties. For example, the constant values will make covariance matrices singular for the LDA and QDA methods.

\section{MACHINE LEARNING METHODS}

Trajectory attributes used to train classifiers are a vehicle's location coordinates and corresponding time stamps. For a pair of intersections (A and B in Figure 1), the training dataset is from one intersection RSU's BSM data and the test dataset is from the other intersection. Let $Y$ represent the ID variable and a $3 \times 1$ vector variable $\mathbf{X}=\left[\begin{array}{lll}X_{1} & X_{2} & X_{3}\end{array}\right]^{T}$ represent the trajectory attributes in which $X_{1}$ and $X_{2}$ are vehicles' local coordinates with the origin defined by the intersection center's coordinates (33). $X_{3}$ represents the trajectory's time stamp. With a trained classifier, the identity of a vehicle is determined by its trajectory points' most classified ID. In this research, an odd number of trajectory points (i.e. 5 trajectory points) is used when the trained model is applied to the test dataset.

\section{Logistic Regression}

LR models the boundary between two trajectory attributes using linear functions with the logit transformation:

$$
\log \frac{\operatorname{Pr}(Y=i \mid \mathbf{X}=\boldsymbol{x})}{\operatorname{Pr}(Y=K \mid \mathbf{X}=\boldsymbol{x})}=\beta_{i 0}+\boldsymbol{\beta}_{i}^{T} \boldsymbol{x}
$$


In which $i=1, \cdots, K-1 . K$ represents the order of ID in the sample. Denote the parameter vector $\boldsymbol{\theta}=\left\{\beta_{10}, \boldsymbol{\beta}_{1}, \cdots, \beta_{(K-1) 0}, \boldsymbol{\beta}_{K-1}\right\}$. Given a trajectory attribute, the probability of being ID $i$ is:

$$
p_{i}(\boldsymbol{x})=\operatorname{Pr}(Y=i \mid \boldsymbol{x})=\left\{\begin{array}{cc}
\frac{\exp \left(\beta_{i 0}+\boldsymbol{\beta}_{i}{ }^{T} \boldsymbol{x}\right)}{1+\sum_{j=1}^{K-1} \exp \left(\beta_{j 0}+\boldsymbol{\beta}_{j}{ }^{T} \boldsymbol{x}\right)} & i=1, \cdots K-1 \\
\frac{1}{1+\sum_{j=1}^{K-1} \exp \left(\beta_{j 0}+\boldsymbol{\beta}_{j}{ }^{T} \boldsymbol{x}\right)} & i=K
\end{array}\right.
$$

The parameter estimation is based on MLE and the likelihood is $l(\theta)=\sum_{v=1}^{n} \log p_{y_{v}}\left(\boldsymbol{\theta} ; \boldsymbol{x}_{v}\right)$, in which $n$ is the total number of trajectory attributes in the training set. Since the likelihood is of a complex form, it can be solved by the traditional numerical method Newton-Raphson algorithm (34). Given an unknown vehicle's trajectory attribute $\boldsymbol{x}$, the assigned ID is:

$$
I D=\arg \max _{i} p_{i}(\boldsymbol{x})
$$

\section{Linear Discriminant Analysis}

The LDA is another popular linear classification model. The assumption is that each trajectory's composing point density is multivariate Normal $N\left(\boldsymbol{\mu}_{i}, \boldsymbol{\Sigma}\right)$ with the same covariance matrix $\boldsymbol{\Sigma}$. Let $\pi_{i}$ be the prior probability of trajectory $i \quad(i=1, \cdots, K)$ and $g_{i}(\boldsymbol{x})$ be the ith trajectoryconditional density. Then the posterior ID probability is $\operatorname{Pr}(Y=i \mid \boldsymbol{x})=g_{i}(\boldsymbol{x}) \pi_{i} / \sum_{j=1}^{K} g_{j}(\boldsymbol{x}) \pi_{j}$. The discriminant function (Equation (4)) for each trajectory ID is obtained using $\log \{\operatorname{Pr}(Y=i \mid \boldsymbol{x}) / \operatorname{Pr}(Y=j \mid \boldsymbol{x})\}$ and some simple algebra.

$$
f_{i}(\boldsymbol{x})=\boldsymbol{x}^{T} \boldsymbol{\Sigma}^{-1} \boldsymbol{\mu}_{i}-\frac{1}{2} \boldsymbol{\mu}_{i}^{T} \boldsymbol{\Sigma}^{-1} \boldsymbol{\mu}_{i}+\log \pi_{i}
$$

The parameter estimation is defined by Equation (5-8)

$$
\begin{aligned}
& \hat{\pi}_{i}=\frac{n_{i}}{n} \\
& \hat{\boldsymbol{\mu}}_{i}=\sum_{i=1}^{K} \frac{\boldsymbol{x}_{i}}{n_{i}} \\
& \hat{\Sigma}=\sum_{i=1}^{K} \frac{n_{i}-1}{\sum_{j=1}^{K}\left(n_{j}-1\right)} S_{i}
\end{aligned}
$$




$$
S_{i}=\frac{1}{n_{i}-1} \sum_{Y_{v}=i}\left(\boldsymbol{x}_{v}-\hat{\boldsymbol{\mu}}_{i}\right)\left(\boldsymbol{x}_{v}-\hat{\boldsymbol{\mu}}_{i}\right)^{T}
$$

In which $S_{i}$ is $i t h$ trajectory attributes' covariance matrix. Given an unknown vehicle's trajectory attribute $\boldsymbol{x}$, the assigned ID is:

$$
I D=\underset{i}{\arg \max } f_{i}(\boldsymbol{x})
$$

\section{Quadratic Discriminant Analysis}

The QDA model assumptions are similar to LDA's and the only difference is that the trajectories' multivariate Normal $N\left(\boldsymbol{\mu}_{i}, \boldsymbol{\Sigma}_{i}\right)$ densities can have their own covariance matrix $\boldsymbol{\Sigma}_{i}$. The corresponding discriminant function is:

$$
f_{i}(\boldsymbol{x})=-\frac{1}{2} \log \left|\boldsymbol{\Sigma}_{i}\right|-\frac{1}{2}\left(\boldsymbol{x}-\boldsymbol{\mu}_{i}\right)^{T} \boldsymbol{\Sigma}_{i}^{-1}\left(\boldsymbol{x}-\boldsymbol{\mu}_{i}\right)+\log \pi_{i}
$$

The parameter estimation procedure (Equation (5-7)) is applied to QDA, but the covariance matrix estimation is different:

$$
\hat{\boldsymbol{\Sigma}}_{i}=\frac{1}{n_{i}-1} \sum_{Y_{v}=i}\left(\boldsymbol{x}_{v}-\hat{\boldsymbol{\mu}}_{i}\right)\left(\boldsymbol{x}_{v}-\hat{\boldsymbol{\mu}}_{i}\right)^{T}
$$

\section{Linear Support Vector Machine}

In the Linear SVM literature, the boundary separating two trajectories is also in the linear form. The decision function is of the form $f(\boldsymbol{x})=\beta_{0}+\boldsymbol{\beta}^{T} \boldsymbol{x}$ and the parameter estimation is based on the idea of maximizing the margin between the two trajectories (35). Solving such a problem is an exercise in the convex optimization. Given that the two trajectories' IDs are coded as -1 and +1 , the popular setup is:

$$
\begin{gathered}
\min _{\beta_{0}, \boldsymbol{\beta}} \frac{1}{2}\|\boldsymbol{\beta}\|^{2}+C \sum_{i=1}^{N} \xi_{i} \\
\text { subject to } \quad \xi_{i} \geq 0, y_{i}\left(\boldsymbol{x}_{i}^{T} \boldsymbol{\beta}+\beta_{0}\right) \geq 1-\xi_{i}, \forall i
\end{gathered}
$$

In which $\xi_{i}$ is the non-negative slack variable allowing trajectory attributes to be on wrong side of the decision boundary. $N$ is the total number of two training trajectories' attributes. $C$ is a cost parameter that controls the overlap and is decided by a 10 -fold Cross Validation (36). A quadratic programming solution of $\boldsymbol{\beta}$ and $\beta_{0}$ using Lagrange multipliers was introduced by (37). 
The solution for $\boldsymbol{\beta}$ has the form $\hat{\boldsymbol{\beta}}=\sum_{i=1}^{N} \hat{\alpha}_{i} y_{i} \boldsymbol{x}_{i}$ in which $\hat{\alpha}_{i}$ is a positive Lagrange multiplier and the resulting decision function is expressed by Equation (14) in which $\langle\cdot, \cdot\rangle$ is the inner product operator. Given an unknown vehicle's trajectory attribute $\boldsymbol{x}$, the assigned ID is indicated by Equation (15).

$$
\begin{gathered}
\hat{f}(\boldsymbol{x})=\hat{\beta}_{0}+\sum_{i=1}^{N} \hat{\alpha}_{i} y_{i}\left\langle\boldsymbol{x}, \boldsymbol{x}_{i}\right\rangle \\
I D=\operatorname{sign}[\hat{f}(\boldsymbol{x})]
\end{gathered}
$$

For $m \geq 2$ vehicle ID matching problems, a 'one against one' approach is adopted and $m(m-1) / 2$ binary decision functions are estimated. The final decision is made based on the most identified ID by all the binary classifiers.

\section{Nonlinear Support Vector Machine}

The nonlinear SVM allows more general decision surfaces than linear boundaries of the linear SVM. This flexibility comes from the enlarged feature space consisting of the expanded basis function $h(\boldsymbol{x})=\left(h_{1}(\boldsymbol{x}), h_{2}(\boldsymbol{x}), \cdots, h_{M}(\boldsymbol{x})\right)$. The corresponding estimated decision function is indicated by Equation (16).

$$
\hat{f}(\boldsymbol{x})=\hat{\beta}_{0}+\sum_{i=1}^{N} \hat{\alpha}_{i} y_{i}\left\langle h(\boldsymbol{x}), h\left(\boldsymbol{x}_{i}\right)\right\rangle
$$

In which the inner product of expanded basis $\left\langle h(\boldsymbol{x}), h\left(\boldsymbol{x}_{i}\right)\right\rangle$ is further defined by the kernel function $K\left(\boldsymbol{x}, \boldsymbol{x}_{i}\right)$. Two popular choices for the $K$ function are implemented in this research, the polynomial kernel function by Equation (17) and the Gaussian kernel function by Equation (18). The parameter $d$ and $\sigma$ are the tuning parameters determined using a 10-fold Cross Validation. Finally, the ID matching rule is same as formula (14).

$$
\begin{aligned}
& K\left(\boldsymbol{x}, \boldsymbol{x}_{i}\right)=\left(1+\left\langle\boldsymbol{x}, \boldsymbol{x}_{i}\right\rangle\right)^{d} \\
& K\left(\boldsymbol{x}, \boldsymbol{x}_{i}\right)=\exp \left(-\left\|\boldsymbol{x}-\boldsymbol{x}_{i}\right\|^{2} / \sigma\right)
\end{aligned}
$$

\section{K Nearest Neighbor}

The KNN classifier needs no model to be fit. The idea of this matching mechanism is to assign the ID to vehicle trajectory attribute based on the closest $K$ training examples in the spatial and temporal space. The attribute $\boldsymbol{x}$ is assigned to the ID most common amongst its $\mathrm{K}$ nearest neighbors. The metric to measure distances between attributes is the Euclidean distance. The tuning parameter $\mathrm{K}$ is decided by a 10 -fold Cross Validation. 


\section{RESULT}

\section{Experiment Design}

To investigate the matching accuracy, a one-hour simulation data was collected. Reasons for using simulation data are: first, there are only a few OBUs (less than 10) in deployment. The small quantity of OBUs limits the experimental capability to investigate basic scenario impacts on matching accuracy. Second, using simulation data renders us able to collect BSM data across all kinds of condition combinations, some of which are rarely seen in real world (e.g. 100\% CV market penetration rate)

Besides the method factor, other factors include the traffic volume, CV market penetration rate, number of vehicles to be matched and the location. Among those four factors, the traffic volume, $\mathrm{CV}$ market penetration and number of vehicles to be matched are treated as random factors, indicating that if the experiment is conducted again, the treatment levels may be different. Three traffic volume levels are selected randomly: $200 \mathrm{veh} / \mathrm{h}$ on the main street and $100 \mathrm{veh} / \mathrm{h}$ on side streets for the low volume, $400 \mathrm{veh} / \mathrm{h}$ on the main street and $200 \mathrm{veh} / \mathrm{h}$ on side streets for the medium volume, $800 \mathrm{veh} / \mathrm{h}$ on the main street and $400 \mathrm{veh} / \mathrm{h}$ on side street for the high volume. Different CV market penetration rates are: $20 \%, 60 \%$ and $100 \%$. Six numbers of vehicles are matched: 2, 5, 10,30, 60 and 100. The method and the location are regarded as fixed factors. Four pairs of intersections are selected from the MMITTS CV test corridor at Anthem, Arizona (Figure 2).

Due to the traffic volume limitations, the number of vehicles to be matched and the traffic volume levels cannot be fully crossed in this experiment. For the low traffic volume case, only 2 , 5, 10 and 30 vehicles are used for ID matching. For the medium traffic volume, additional 60 vehicles are used. For the high traffic volume case, all the vehicle number levels are used. For some vehicle number and traffic volume combinations, there is no result data collected. This characteristic makes this experiment an unbalanced one. Vehicles' trajectory data is collected through the SIL technique in which the RSU applications collect BSMs sent by CVs in the VISSIM simulation environment (31). For each combination of the four factors, five replications of matching experiments are conducted by different random seeds in the $\mathrm{R}$ software environment.

The total number of matching records is 6300 and the average mis-matching rates are summarized by factor in Table 1. A first look at the table reveals that the Logistic Regression has the worst performance among all test methods. The LDA, QDA and Linear SVM have the relatively smallest mis-matching rates. More vehicles lead to higher mis-matching rates. Differences of mis-matching rates exist among subcategories of Location, Market Penetration Rate and Traffic Volume. Due to the complexity and unbalanced characteristics of this experiment, a formal analysis is proposed below.

Since the mis-matched number is a non-negative integer, for the purpose of investigating impacts of associated fixed and random factors, a Poisson mixed regression model is appropriate. The traditional standard Poisson regression usually suffers from the heterogeneity problem in count data. Over dispersion is the major heterogeneity form in Poisson distribution (38). In other words, only the fixed and random factors cannot guarantee to explain all the variations in the data. This extra variation occurred in modeling count data is accounted by an additional random residual term. Equation (19) and (20) are proposed for modeling relations between the count response and the latent variable, which is further predicted by a linear model. 


$$
\begin{gathered}
z_{i} \sim \operatorname{Poi}\left(\lambda_{i}=\exp \left(l_{i}\right)\right) \\
l_{i}=\boldsymbol{x}_{i}^{T} \boldsymbol{\beta}+\boldsymbol{u}_{i}^{T} \boldsymbol{\eta}+e_{i}
\end{gathered}
$$

Where $z_{i}$ represents the ith record of mis-matched number, following the Poisson distribution with the rate $\lambda_{i}$. In the Generalized Linear Model family, the rate $\lambda_{i}$ is related to the latent variable $l_{i}$ given that a canonical log link function is employed (39). Here variables $\boldsymbol{x}$ and $\boldsymbol{\beta}$ are reused. $\boldsymbol{x}_{i}$ is a $10 \times 1$ vector $\boldsymbol{x}_{i}=\left(1, x_{i 1}, x_{i 2}, \ldots, x_{i 9}\right)^{T}$ representing the intercept and fixed factor indicator variables and $\boldsymbol{\beta}$ is a $10 \times 1$ vector $\boldsymbol{\beta}=\left(\beta_{0}, \beta_{1}, \beta_{2}, \ldots, \beta_{9}\right)^{T}$ representing the corresponding effects. $\boldsymbol{u}_{i}$ is a $3 \times 1$ vector $\boldsymbol{u}_{i}=\left(u_{i 1}, u_{i 2}, u_{i 3}\right)^{T}$ representing 3 random factor indicator variables and $\boldsymbol{\eta}$ is a a $3 \times 1$ vector $\boldsymbol{\eta}=\left(\eta_{1}, \eta_{2}, \eta_{3}\right)^{T}$ representing the corresponding effects. $e_{i}$ is the residual term.

The MCMC sampling technique within the Bayesian hierarchical model framework is used for exploring both the fixed and random factors' effects. We follow the work of (40) and make multivariate normal distribution assumption (Equation (21)) for the effect and residual parameters $\left(\boldsymbol{\beta}, \boldsymbol{\eta}\right.$ and $\left.\boldsymbol{e}=\left(e_{1}, \cdots, e_{6300}\right)^{T}\right)$.

$$
\left[\begin{array}{l}
\boldsymbol{\beta} \\
\boldsymbol{\eta} \\
e
\end{array}\right] \sim N\left(\left[\begin{array}{l}
\boldsymbol{\mu} \\
\mathbf{0} \\
\mathbf{0}
\end{array}\right]\left[\begin{array}{lll}
\boldsymbol{B} & \mathbf{0} & \mathbf{0} \\
\mathbf{0} & \boldsymbol{G} & \mathbf{0} \\
\mathbf{0} & \mathbf{0} & \boldsymbol{R}
\end{array}\right]\right)
$$

In which $\boldsymbol{\mu}$ is the prior mean for fixed effects with prior covariance matrix $\boldsymbol{B}$. For random factors and residual term, their prior means are set to 0 and covariances are $\boldsymbol{G}$ and $\boldsymbol{R}$. Parameters can be Gibbs sampled and details can be found in $(40 ; 41)$. Before proceeding to the sampling process, parameter prior distributions should be set up first. For fixed factor, a normal prior is set with $\boldsymbol{\mu}=(0, \cdots, 0)^{T}$ and $\boldsymbol{B}=\operatorname{diag}\left\{10^{8}, \cdots, 10^{8}\right\}$. For random and residual variance components

( $\boldsymbol{G}=\operatorname{diag}\left\{G_{1}, G_{2}, G_{3}\right\}$ and $\left.\boldsymbol{R}=\operatorname{diag}\{r, \cdots, r\}\right)$, inverse gamma distributions are assigned with shape and scale parameters set to 0.001 . The MCMC sample size is $6 \times 10^{6}$ and the first $6 \times 10^{5}$ samples are discarded as the burn-in process. Each parameter sample is collected every 500 iterations for the purpose of being identically and independently distributed.

\section{Model Validation}

The purpose of the Bayesian model validation is to check whether the parameter samples drawn by the MCMC can accurately represent their true posterior distributions.

\section{Visualized Convergence Check}

Parameter Samples of posterior distributions are plotted in Figure 3(a)-3(n). The constant variation and the unchanging mean are good signs of convergence of the sampling process. All the fixed effect and the residual parameters perform well, but the random effect parameter chains (Figure $3(\mathrm{k}), 3(\mathrm{l})$ and $3(\mathrm{~m}))$ for the variances are spiky due to their posterior distributions having long right 
tails. It is helpful to plot them on a log scale to reduce the influence of a few outliers. In Figure $3(\mathrm{o}-\mathrm{q})$, the parameters are behaving better. The visual check implies good convergence of posterior distributions. Additionally, the autocorrelation check (42) is used and shows the evidence of samples being independently distributed.

\section{Gelman-Rubin Test}

The stricter validation approach relies on test statistics and sampling properties. The GelmanRubin test (43) is used. Several independent MCMC experiments are checked whether they converge to the same posterior distribution. By comparing estimated between-chain and with-in chain variances, the sampling chain's convergence is examined. Assuming there are $M$ chains and each has a length of $L$. Let $\hat{\gamma}_{i}$ be the sampled parameter value mean and $\hat{\sigma}_{i}^{2}$ be the sample variance of $i$ th chain. Let $\hat{\gamma}=(1 / M) \sum_{i=1}^{M} \hat{\gamma}_{i}$ be the overall sample posterior mean. Then the between-chain variance is defined by $V_{B}=[L /(M-1)] \sum_{i=1}^{M}\left(\hat{\gamma}_{i}-\hat{\gamma}\right)^{2}$ and the within-chain variance is defined by $V_{W}=(1 / M) \sum_{i=1}^{M} \hat{\sigma}_{i}^{2}$. An unbiased estimator of the marginal posterior variance of $\gamma$ is defined by $\hat{V}=[(L-1) / L] V_{W}+[(M+1) / M L] V_{B}$. In (44), the Potential Scale Reduction Factor (PSRF) test statistic is defined by Equation (22)

$$
R_{c}=\sqrt{\frac{\hat{d}+3}{\hat{d}+1} \frac{\hat{V}}{V_{W}}}
$$

In which $\hat{d}$ is an estimate of degree of freedom of a $t$ distribution. If the $M$ chains have converged to the target posterior distribution, then the value of $R_{c}$ should be close to 1. Practically, if $R_{c}<1.2$ for all parameters, it is safe to say the convergence has been reached. An upper limit (Equation 23) is defined for monitoring the test statistic (43).

$$
R_{u}(\alpha)=\sqrt{\frac{\hat{d}+3}{\hat{d}+1}\left(\frac{L-1}{L} V_{W}+\frac{M+1}{M} q_{1-\alpha / 2}\right)}
$$

In which $q_{1-\alpha / 2}$ is the $(1-\alpha / 2)$ quartile of an $F$ distribution. By calculating the PSRF as the function of sampled parameter values, one can tell when the test statistic is really converged and not just randomly near 1 . Figure 4 shows a plot $\left(M=4, L=6 \times 10^{6}\right)$ of PSRFs (black solid lines) with the upper limits (red dashed lines) at a 95\% confidence level. Note that the Gelman-Rubin convergence test assumes normality for the parameter's marginal posterior distribution. But for the Poisson regression model, there is no available conjugate normal posterior distribution. Therefore, those test statistics are calculated based on data normalized by the logarithm transformation. All parameters' test statistics are approaching 1 quickly, indicating the convergence is achieved. Therefore, the Poisson mixed model parameter samples drawn by the MCMC are validated and are treated as posterior distributions for the further effect analysis. 


\section{Factor Effect Analysis}

After obtaining effect parameters' sampled distributions, their contribution to the mis-matching vehicle rate (per 100 vehicles) should be examined. Medians are used as the estimators. The Highest Posterior Density interval is reported as the Bayesian equivalent Confidence Interval (CI). Table 2 shows the parameter medians, upper and lower limits with $95 \% \mathrm{CI}$ and the effective sample sizes. Effective sample sizes are different due to the MCMC sampling rejection rule (34), if no sample is rejected then the sample size is $\left(6 \times 10^{6}-6 \times 10^{5}\right) / 500=10800$.

The factor effects on mis-matching rates differ among locations and methods. All fixed factors' CIs do not include 0, indicating their impacts are significant. For the default level combination (the Intercept), location Intersection Pair 1 and LR, the mean of the expected mismatching rate is 35 per 100 vehicles $(\exp (3.55) \approx 35)$. Given the matching is conducted in the same intersection pair, all the other matching methods have better performance than LR, since their coefficients' sample medians have negative impacts on the mis-matching rate. Specifically, the Linear SVM $(\exp (3.55-1.78) \approx 5.87)$, the QDA $(\exp (3.55-1.74) \approx 6.11)$ and the LDA $(\exp (3.55-1.69) \approx 6.42)$ have the best top 3 accuracy performances. All the other locations have worse performance than Intersection Pair 1, since their medians all have positive impacts on the mis-matching rate. The performance ranking is consistent with their within distances. For example, Intersection Pair 1 has the shortest within distance and the smallest mis-matching rate if the method is kept unchanged, while the Intersection Pair 2 has the largest within distance and the largest mismatching rate (sample median value 1.24).

For the 3 random factors, their variances on the mis-matching rate are the experiment designer's interest (22), and therefore their specific levels' impacts are not included in the model. The variance components of the traffic volume factor and the market penetration rate factor have the least impacts on the mis-matching rate, since their sample medians are 0.03 and 0.01 . This is suggesting that if the ID matching is conducted in a different traffic volume situation or in a different CV market penetration rate scenario, given other factors are kept unchanged, there will be little difference in the result's accuracy. To the contrary, the variance component of the number of vehicles to be matched factor has a large influence on the mis-matching rate with a median value 0.81 . This is suggesting that conducting the CV ID matching with 10 vehicles will have significant difference in the result's accuracy compared with doing that with 60 vehicles or 100 vehicles. Meanwhile, the residual's variance component is not negligible (sample median value 0.17), indicating that the data is not strictly Poisson distributed but with slight over dispersion.

\section{CONCLUSION}

The advantages of proposed methods include 3 aspects: First, this paper demonstrates how to use the structured $\mathrm{CV}$ data rather than the unstructured image data for vehicle reidentification. Seven popular machine learning classification methods are implemented using the vehicle's trajectory attributes. Second, potential factors impacting matching accuracy are systematically investigated. A Poisson mixed regression model is proposed to analyze the effects of potential factors on matching accuracy. The analysis is conducted based on the Bayesian inference framework. The experiment result shows that the Linear SVM, the QDA and the LDA are the 3 best techniques out of all the candidate machine learning methods discussed in this paper for CV ID matching. Additionally, the location factor and the number of vehicles to be matched factor have significant 
impacts on matching accuracy, while the traffic volume factor and the CV market penetration factor are not considered significant. Third, this research work can help with future CV based research and also serve as an evidence for public concern. Note that the smallest average mismatching rate is $14 \%$ achieved by the 3 mentioned machine learning techniques (Table 1). This possibility of using trajectory data to reidentify connected vehicle ID with high accuracy renders researchers capable of assessing performance of $\mathrm{CV}$ based applications in the situation where vehicle IDs are changing frequently. However, securer protection mechanisms for trajectory data should be investigated and the access to trajectory data should be carefully controlled.

One limitation when using simulation data is assuming that there are no new vehicles appearing in the test data set. For instance, when a new vehicle enters the traffic stream from a parking lot between intersections, the mis-matching rate will likely increase. One mitigation measure to reduce the influence of newly appeared vehicles is to use static vehicle properties to do filtering. For example, if only 10 sedan vehicles appear in the first RSU range, then any other type of vehicle appearing at the next RSU must be a new vehicle which does not need ID matching. But such a mechanism cannot ensure new vehicles are filtered out every time given that they may have the same static properties as existing vehicles. The ultimate solution must be considered in the classification method itself. The Zero Shot Learning (ZSL) techniques (45) are promising in this regard. Future work can include such considerations and other matching techniques. 
The authors confirm contribution to the paper as follows: study conception and design: Zuoyu Miao, K. Larry Head, Byungho Beak; data collection: Zuoyu Miao, K. Larry Head, Byungho Beak; analysis and interpretation of results: Zuoyu Miao, K. Larry Head, Byungho Beak; draft manuscript preparation: Zuoyu Miao, K. Larry Head, Byungho Beak. All authors reviewed the results and approved the final version of the manuscript.

\section{REFERENCES}

1. FHWA. FHWA Announces Vehicle-to-Infrastructure Guidance. Federal Highway Administration. https://www.its.dot.gov/v2i/. Accessed Jan. 19, 2017.

2. Juliana Gruenwald Henderson, K. J. Connected Cars: Privacy, Security Issues Related to Connected, Automated Vehicles. https://www.ftc.gov/news-events/eventscalendar/2017/06/connected-cars-privacy-security-issues-related-connected.

3. Zeng, N., and J. D. Crisman. Vehicle matching using color.In Intelligent Transportation System, 1997. ITSC'97., IEEE Conference on, IEEE, 1997. pp. 206-211.

4. Kogut, G. T., and M. Trivedi. Maintaining the identity of multiple vehicles as they travel through a video network.In Intelligent Transportation Systems, 2001. Proceedings. 2001 IEEE, IEEE, 2001. pp. 756-761.

5. MacCarley, C. A. Video-based vehicle signature analysis and tracking system phase 2: algorithm development and preliminary testing. California Partners for Advanced Transit and Highways (PATH), 2001.

6. Wang, S., L. Cui, D. Liu, R. Huck, P. Verma, J. J. Sluss, and S. Cheng. Vehicle identification via sparse representation. IEEE Transactions on intelligent transportation systems, Vol. 13, No. 2 , 2012, pp. 955-962.

7. Li, P., and R. R. Souleyrette. A Generic Approach to Estimate Freeway Traffic Time Using Vehicle ID - Matching Technologies. Computer - Aided Civil and Infrastructure Engineering, Vol. 31, No. 5, 2016, pp. 351-365.

8. Haghani, A., M. Hamedi, K. Sadabadi, S. Young, and P. Tarnoff. Data collection of freeway travel time ground truth with bluetooth sensors. Transportation Research Record: Journal of the Transportation Research Board, No. 2160, 2010, pp. 60-68.

9. Barceló, J., L. Montero, L. Marqués, and C. Carmona. Travel time forecasting and dynamic origin-destination estimation for freeways based on bluetooth traffic monitoring. Transportation Research Record: Journal of the Transportation Research Board, No. 2175, 2010, pp. 19-27.

10. Brennan Jr, T. M., J. M. Ernst, C. M. Day, D. M. Bullock, J. V. Krogmeier, and M. Martchouk. Influence of vertical sensor placement on data collection efficiency from bluetooth MAC address collection devices. Journal of Transportation Engineering, Vol. 136, No. 12, 2010, pp. 11041109 .

11. Hainen, A., J. Wasson, S. Hubbard, S. Remias, G. Farnsworth, and D. Bullock. Estimating route choice and travel time reliability with field observations of Bluetooth probe vehicles.

Transportation Research Record: Journal of the Transportation Research Board, No. 2256, 2011, pp. 43-50.

12. Quayle, S., P. Koonce, D. DePencier, and D. Bullock. Arterial performance measures with media access control readers: Portland, Oregon, pilot study. Transportation Research Record: Journal of the Transportation Research Board, No. 2192, 2010, pp. 185-193.

13. Richardson, J., B. Smith, M. Fontaine, and S. Turner. Network stratification method by travel time variation. Transportation Research Record: Journal of the Transportation Research Board, No. 2256, 2011, pp. 1-9.

14. Hofleitner, A., R. Herring, and A. Bayen. Arterial travel time forecast with streaming data: A hybrid approach of flow modeling and machine learning. Transportation Research Part B:

Methodological, Vol. 46, No. 9, 2012, pp. 1097-1122. 
15. Charbonnier, S., A.-C. Pitton, and A. Vassilev. Vehicle re-identification with a single magnetic sensor.In Instrumentation and Measurement Technology Conference (I2MTC), 2012 IEEE International, IEEE, 2012. pp. 380-385.

16. Sanchez, R. O., C. Flores, R. Horowitz, R. Rajagopal, and P. Varaiya. Arterial travel time estimation based on vehicle re-identification using magnetic sensors: Performance analysis.In Intelligent Transportation Systems (ITSC), 2011 14th International IEEE Conference on, IEEE, 2011. pp. 997-1002.

17. Kwong, K., R. Kavaler, R. Rajagopal, and P. Varaiya. Arterial travel time estimation based on vehicle re-identification using wireless magnetic sensors. Transportation Research Part C:

Emerging Technologies, Vol. 17, No. 6, 2009, pp. 586-606.

18. Kavaler, R., K. Kwong, A. Raman, P. Varaiya, and D. Xing. Arterial performance measurement system with wireless magnetic sensors.In ICTIS 2011: Multimodal Approach to Sustained Transportation System Development: Information, Technology, Implementation, 2011. pp. 377385.

19. Sivaraman, S., and M. M. Trivedi. Looking at vehicles on the road: A survey of vision-based vehicle detection, tracking, and behavior analysis. IEEE Transactions on intelligent

transportation systems, Vol. 14, No. 4, 2013, pp. 1773-1795.

20. Searle, S. R., and M. H. Gruber. Linear models. John Wiley \& Sons, 2016.

21. Speed, F. M., R. R. Hocking, and O. Hackney. Methods of analysis of linear models with unbalanced data. Journal of the American Statistical Association, Vol. 73, No. 361, 1978, pp. $105-112$.

22. Montgomery, D. C. Design and analysis of experiments. John Wiley \& Sons, 2017.

23. Box, G. E., and D. R. Cox. An analysis of transformations. Journal of the Royal Statistical Society. Series B (Methodological), 1964, pp. 211-252.

24. Corbeil, R. R., and S. R. Searle. Restricted maximum likelihood (REML) estimation of variance components in the mixed model. Technometrics, Vol. 18, No. 1, 1976, pp. 31-38.

25. Schall, R. Estimation in generalized linear models with random effects. Biometrika, Vol. 78, No. 4, 1991, pp. 719-727.

26. Wolfinger, R., and M. O'connell. Generalized linear mixed models a pseudo-likelihood approach. Journal of statistical Computation and Simulation, Vol. 48, No. 3-4, 1993, pp. 233-243.

27. Breslow, N. E., and D. G. Clayton. Approximate inference in generalized linear mixed models. Journal of the American Statistical Association, Vol. 88, No. 421, 1993, pp. 9-25.

28. Pinheiro, J. C., and E. C. Chao. Efficient Laplacian and adaptive Gaussian quadrature algorithms for multilevel generalized linear mixed models. Journal of Computational and Graphical Statistics, Vol. 15, No. 1, 2006, pp. 58-81.

29. Bolker, B. M., M. E. Brooks, C. J. Clark, S. W. Geange, J. R. Poulsen, M. H. H. Stevens, and J.S. S. White. Generalized linear mixed models: a practical guide for ecology and evolution. Trends in ecology \& evolution, Vol. 24, No. 3, 2009, pp. 127-135.

30. Gilks, W. R., S. Richardson, and D. J. Spiegelhalter. Introducing markov chain monte carlo. Markov chain Monte Carlo in practice, Vol. 1, 1996, p. 19.

31. University of Arizona, University of California PATH Program, Savari Networks Inc., Econolite. Multi-Modal Intelligent Traffic Signal System - Phase II: System Development, Deployment and Field Test Center for Transportation Studies. http://www.cts.virginia.edu/cvpfs research/.

32. Kenney, J. B. Dedicated short-range communications (DSRC) standards in the United States. Proceedings of the IEEE, Vol. 99, No. 7, 2011, pp. 1162-1182.

33. Barth, M., and J. A. Farrell. The Global Positioning System \& Inertial Navigation. McGrawHill, Vol. 8, 1999, pp. 21-56.

34. Rizzo, M. L. Statistical computing with R. CRC Press, 2007.

35. Vapnik, V. The nature of statistical learning theory. Springer science \& business media, 2013.

36. Geisser, S. The predictive sample reuse method with applications. Journal of the American Statistical Association, Vol. 70, No. 350, 1975, pp. 320-328. 
37. Friedman, J., T. Hastie, and R. Tibshirani. The elements of statistical learning. Springer series in statistics Springer, Berlin, 2001.

38. Cameron, A. C., and P. K. Trivedi. Regression analysis of count data. Cambridge university press, 2013.

39. Nelder, J. A., and R. J. Baker. Generalized linear models. Wiley Online Library, 1972.

40. Hadfield, J. D. MCMC methods for multi-response generalized linear mixed models: the MCMCglmm R package. Journal of statistical software, Vol. 33, No. 2, 2010, pp. 1-22.

41. García-Cortés, L. A., and D. Sorensen. Alternative implementations of Monte Carlo EM algorithms for likelihood inferences. Genetics Selection Evolution, Vol. 33, No. 4, 2001, p. 443.

42. Cryer, J. D., and K.-s. Chan. Time series analysis with applications in R.In Springer texts in statistics, Springer, New York, 2008. pp. 1 online resource (xiii, 491 p.).

43. Gelman, A., and D. B. Rubin. Inference from iterative simulation using multiple sequences. Statistical science, 1992, pp. 457-472.

44. Brooks, S. P., and A. Gelman. General methods for monitoring convergence of iterative simulations. Journal of Computational and Graphical Statistics, Vol. 7, No. 4, 1998, pp. 434-455.

45. Xian, Y., B. Schiele, and Z. Akata. Zero-Shot Learning-The Good, the Bad and the Ugly. arXiv preprint arXiv:1703.04394, 2017. 


\section{LIST OF FIGURES}

FIGURE 1 Vehicle Trajectories

FIGURE 2 CV Test Corridor

FIGURE 3 Time Series of Parameter Traces

FIGURE 4 PSRF Plot

TABLE 1 Average Mis-Matching Rates

TABLE 2 Posterior Distribution Summary 


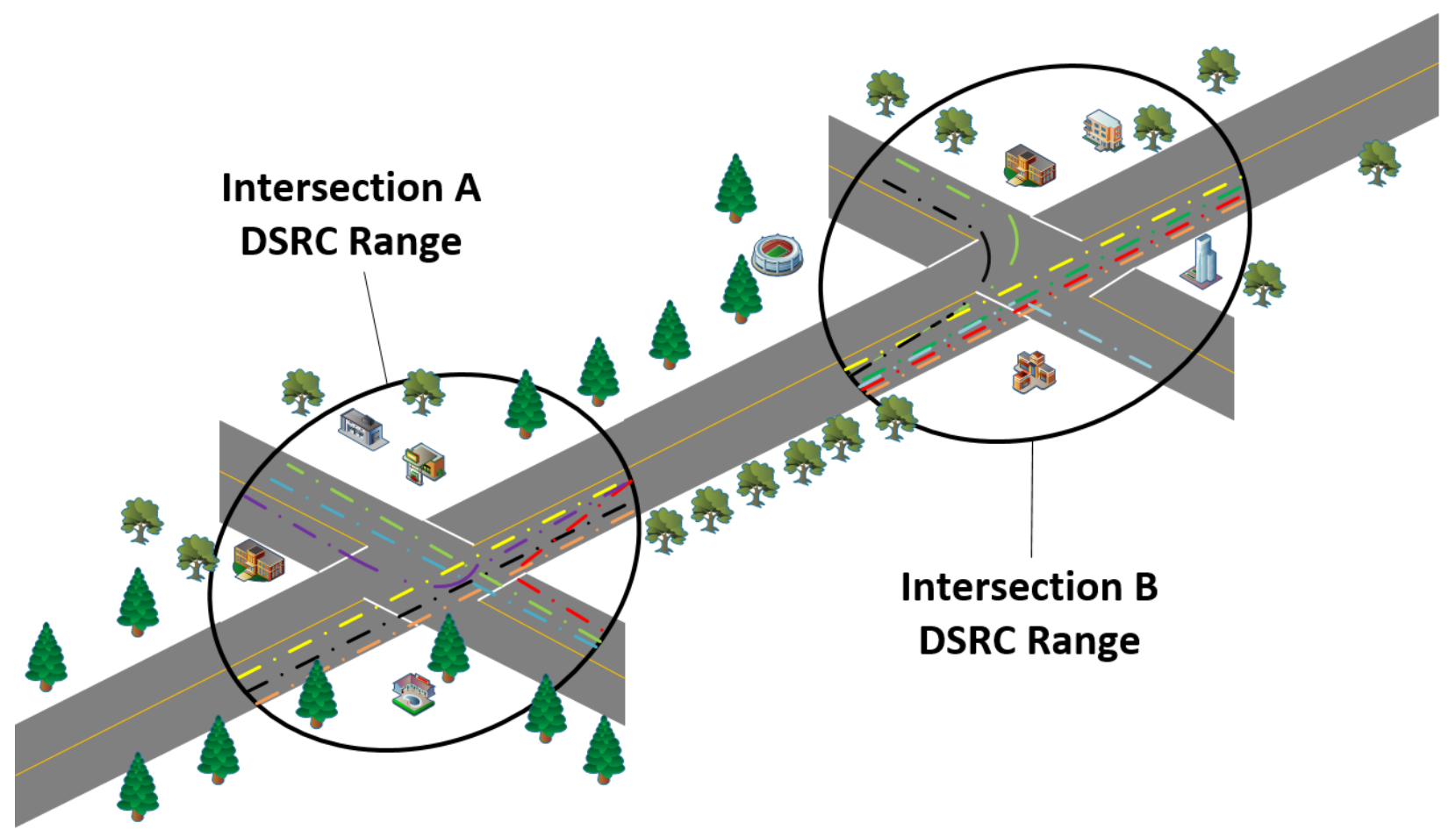

FIGURE 1 Vehicle Trajectories 


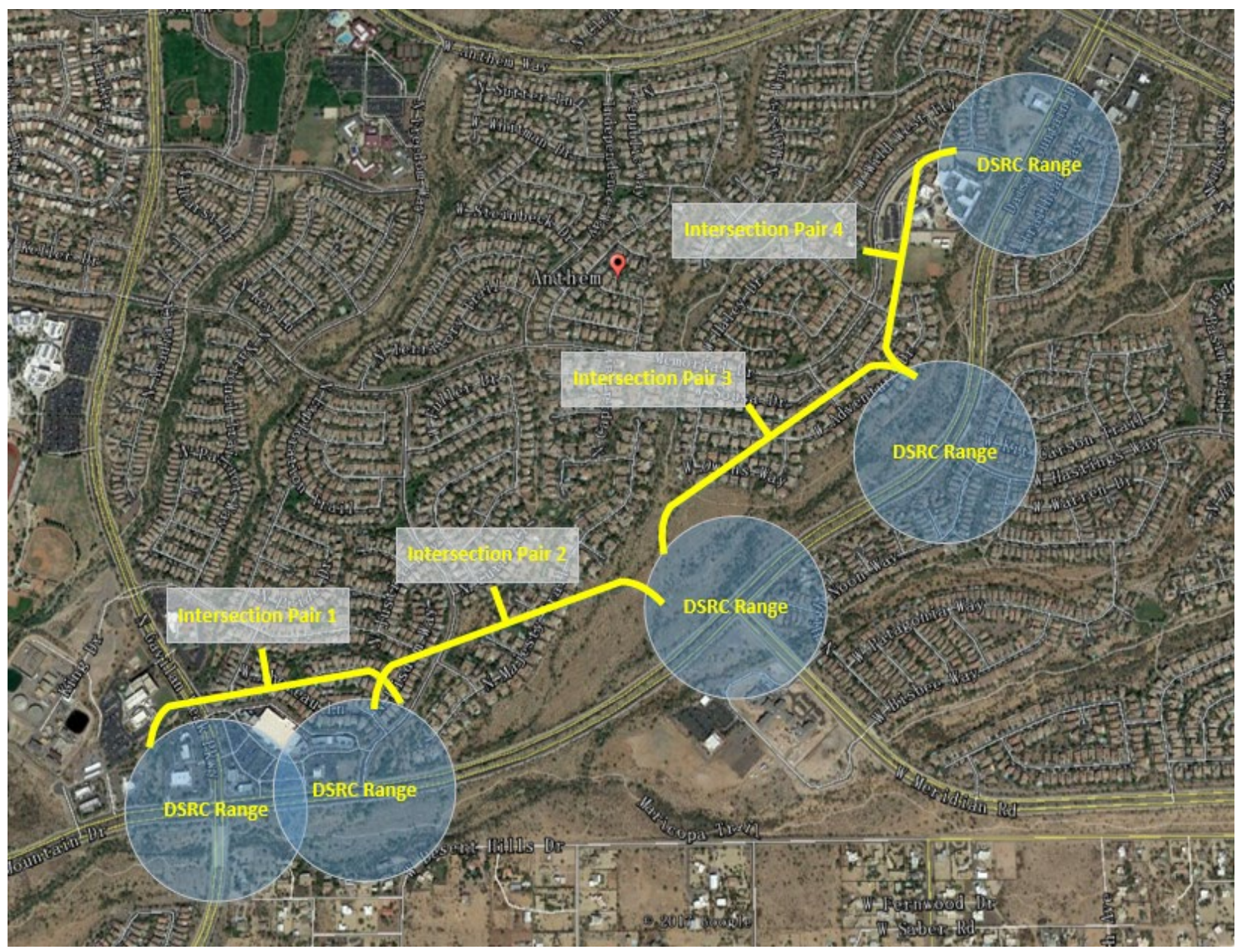

FIGURE 2 CV Test Corridor 


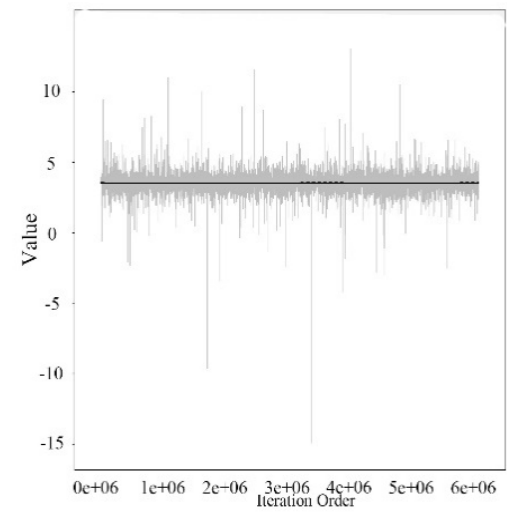

(a) Intercept

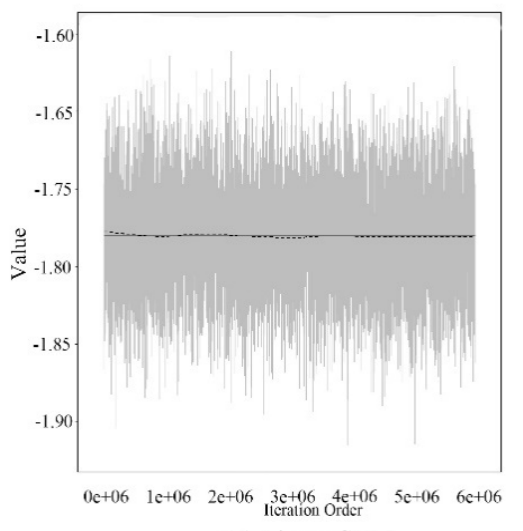

(d) Linear SVM

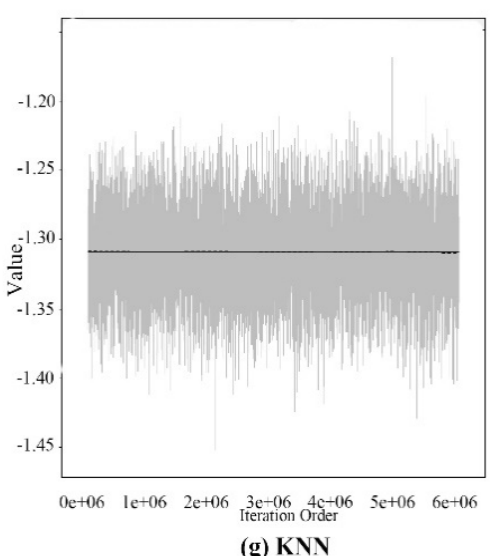

(g) $\mathrm{KNN}$

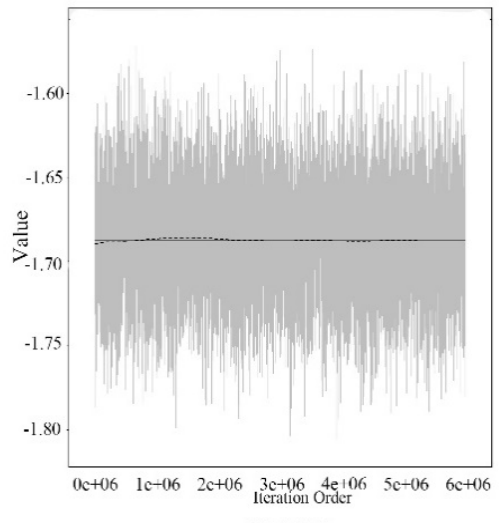

(b) LDA

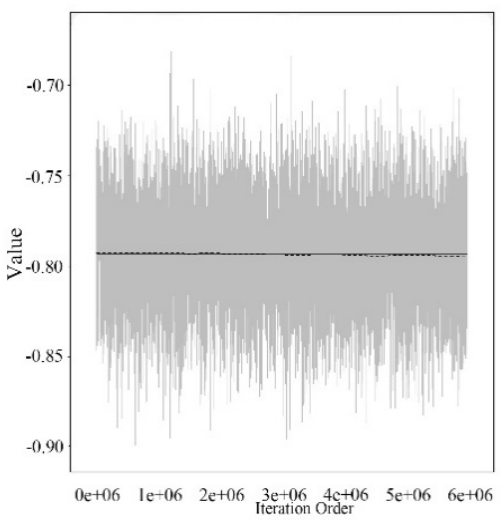

(e) Guassian Kernel SVM

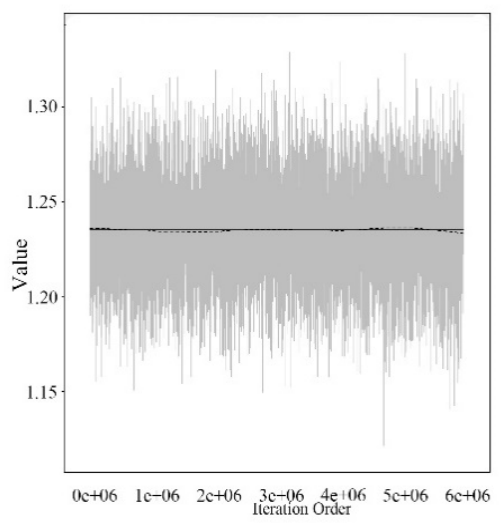

(h)Intersection Pair 2

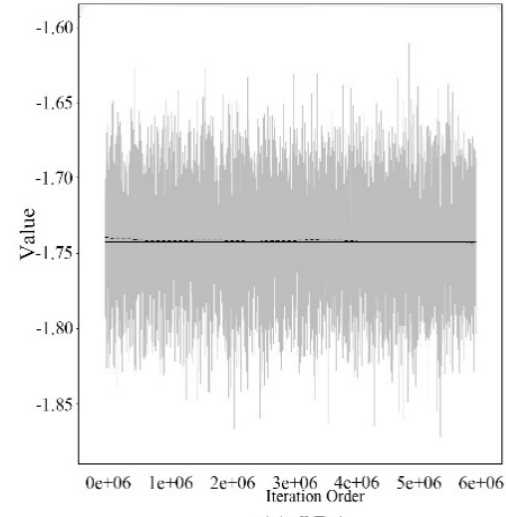

(c) QDA

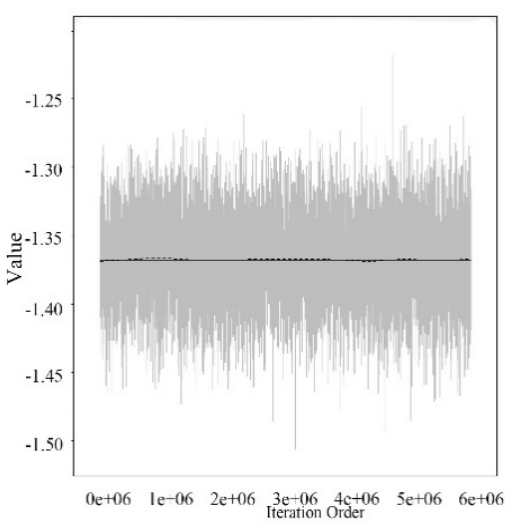

(f) Polynomial Kernel SVM

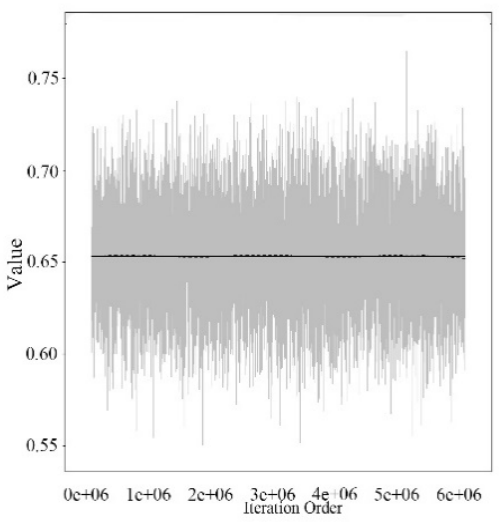

(i)Intersection Pair 3 


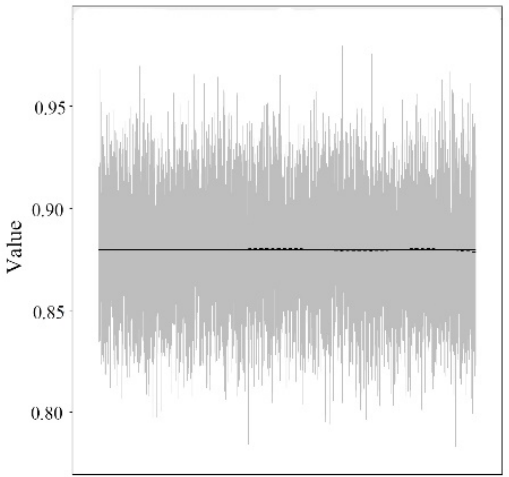

$0 \mathrm{c}+06 \quad 1 \mathrm{c}+06 \quad 2 \mathrm{c}+06 \quad \begin{gathered}3 \mathrm{c}+06 \\ \text { Iteration Order }\end{gathered} \begin{array}{cccc}4 \mathrm{e}+06 & 5 \mathrm{c}+06 & 6 \mathrm{c}+06\end{array}$

(j) Intersection Pair 4

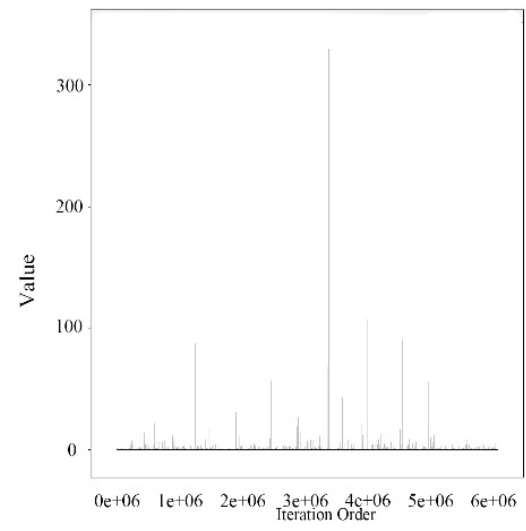

(m) Market Penetration Rate

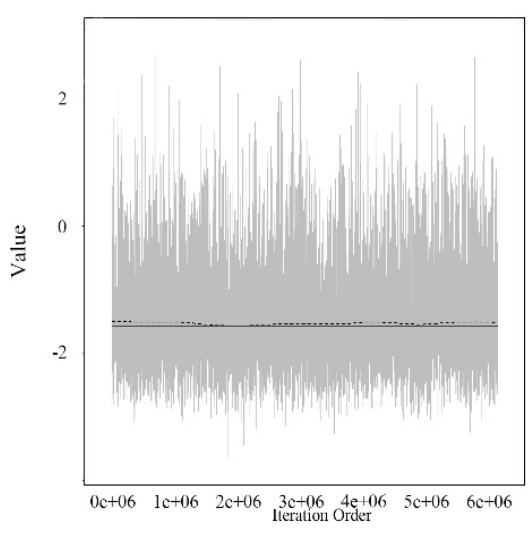

(p) Traffic Volume (Rescaled)

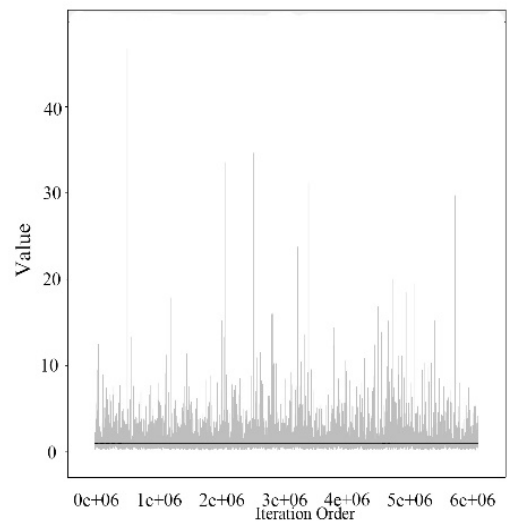

(k) Number of Vehicles to Be Matched

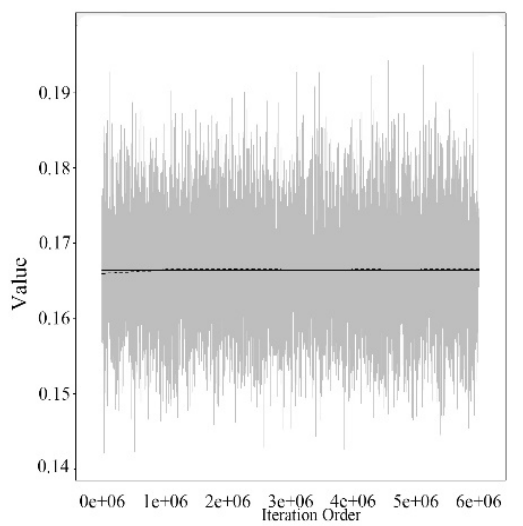

(n) Residual

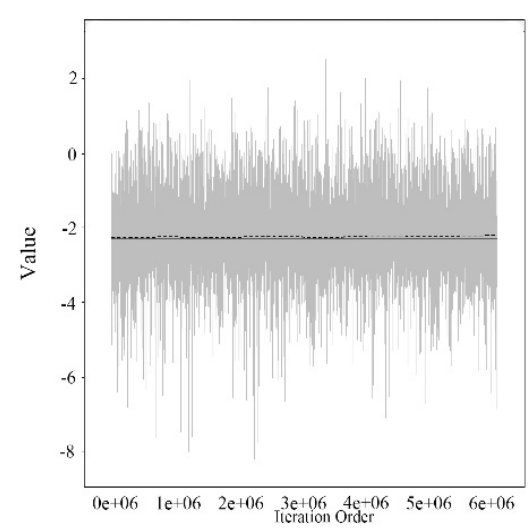

(q) Market Penetration Rate (Rescaled)

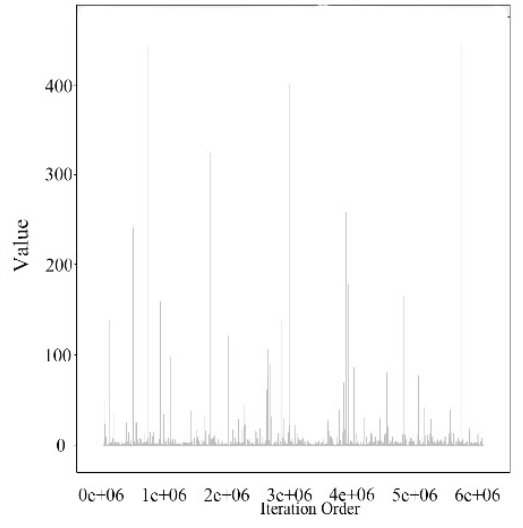

(I) Traffic Volume

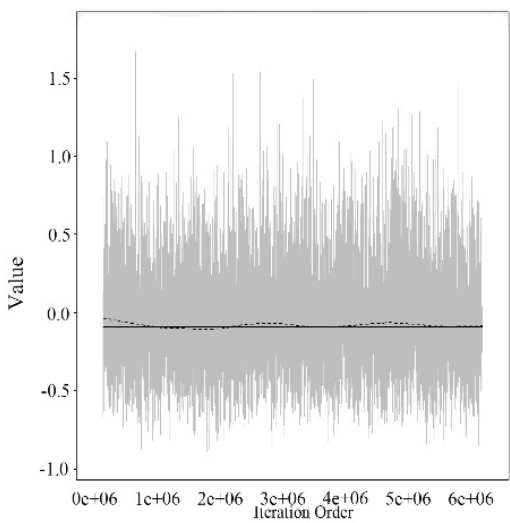

(o) Number of Vehicles to Be

Matched (Rescaled)

FIGURE 3 Time Series of Parameter Traces 

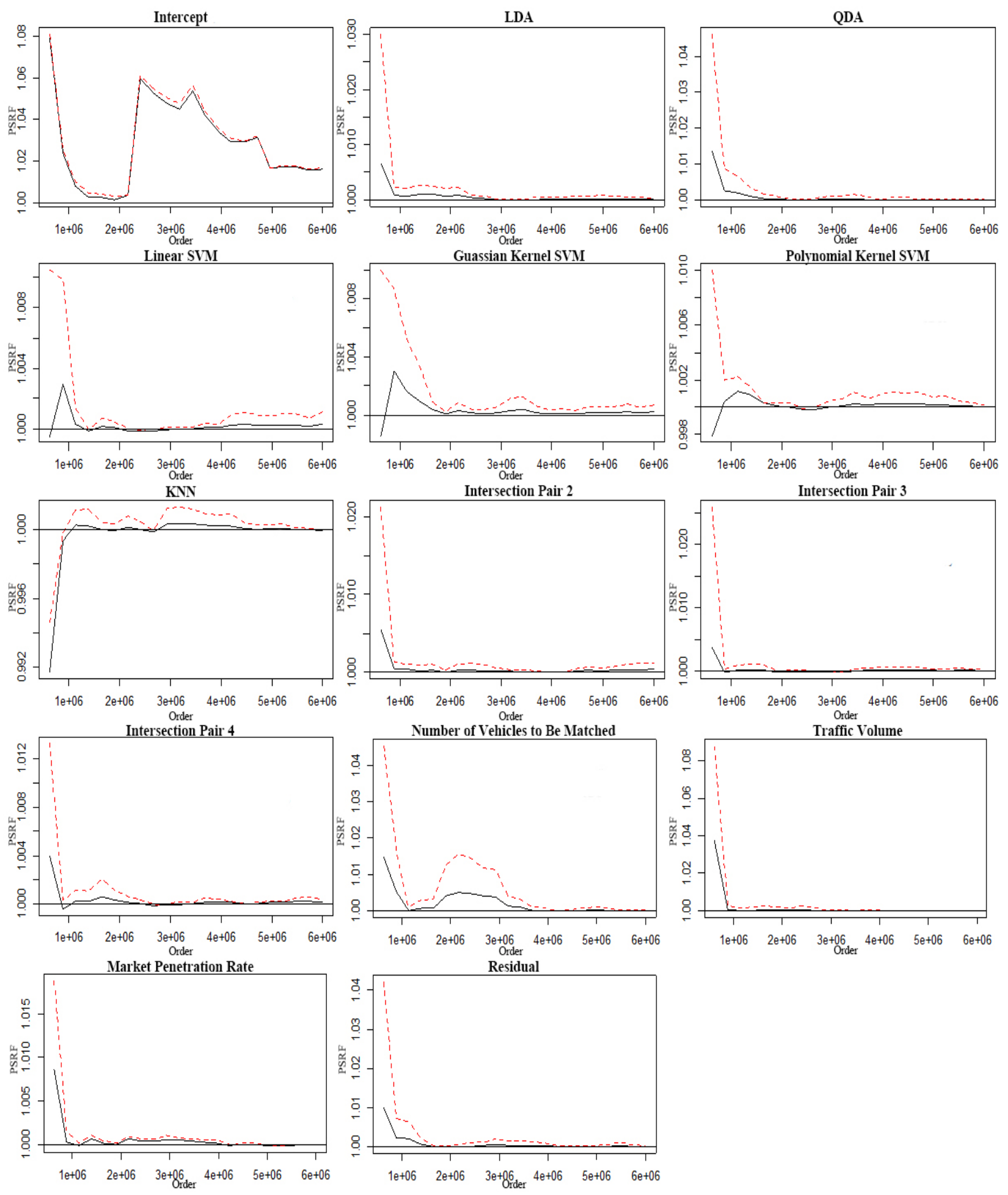

FIGURE 4 PSRF Plot 
TABLE 1 Average Mis-Matching Rates

\begin{tabular}{|c|c|c|c|c|c|c|c|c|c|}
\hline Method & Average & $\begin{array}{c}\text { Numer of } \\
\text { Vehicles to Be } \\
\text { Mateched }\end{array}$ & Average & Location & Average & $\begin{array}{c}\text { Market } \\
\text { Penetration } \\
\text { Rate }\end{array}$ & Average & $\begin{array}{c}\text { Traffic } \\
\text { Volume }\end{array}$ & Average \\
\hline LR & 0.82 & $2 \mathrm{Veh}$ & 0.10 & $\begin{array}{c}\text { Intersection } \\
\text { Pair } 1\end{array}$ & 0.17 & $20 \%$ & 0.30 & Low & 0.23 \\
\hline LDA & 0.14 & 5 Veh & 0.17 & $\begin{array}{c}\text { Intersection } \\
\text { Pair } \mathbf{2}\end{array}$ & 0.44 & $60 \%$ & 0.31 & Medium & 0.30 \\
\hline QDA & 0.14 & 10 Veh & 0.26 & $\begin{array}{c}\text { Intersection } \\
\text { Pair } 3\end{array}$ & 0.27 & $100 \%$ & 0.31 & High & 0.36 \\
\hline Linear SVM & 0.14 & $30 \mathrm{Veh}$ & 0.41 & $\begin{array}{l}\text { Intersection } \\
\text { Pair } 4\end{array}$ & 0.33 & & & & \\
\hline $\begin{array}{c}\text { Guassian } \\
\text { Kernel SVM }\end{array}$ & 0.45 & $60 \mathrm{Veh}$ & 0.53 & & & & & & \\
\hline $\begin{array}{l}\text { Polynomial } \\
\text { Kernel SVM }\end{array}$ & 0.22 & $100 \mathrm{Veh}$ & 0.63 & & & & & & \\
\hline KNN & 0.21 & & & & & & & & \\
\hline
\end{tabular}


TABLE 2 Posterior Distribution Summary

\begin{tabular}{|c|c|c|c|c|c|c|}
\hline Factor Type & Factor & Factor Level & Sample Median & Lower $95 \%$ CI & Upper $95 \%$ CI & Effective Sample Size \\
\hline \multirow{10}{*}{ Fixed } & Intercept & Intersection Pair 1+LR & 3.55 & 2.33 & 4.67 & 10513 \\
\hline & \multirow{6}{*}{ Method } & LDA & -1.69 & -1.75 & -1.62 & 10800 \\
\hline & & QDA & -1.74 & -1.80 & -1.68 & 10800 \\
\hline & & Line ar SVM & -1.78 & -1.85 & -1.71 & 9507 \\
\hline & & \multirow{3}{*}{$\begin{array}{c}\text { Gussian Kernel SVM } \\
\text { Polynomial Kernel } \\
\text { SVM } \\
\text { KNN }\end{array}$} & -0.79 & -0.85 & -0.74 & 10800 \\
\hline & & & -1.37 & -1.43 & -1.31 & 9014 \\
\hline & & & -1.31 & -1.37 & -1.25 & 10334 \\
\hline & \multirow{3}{*}{ Location } & Intersection Pair 2 & 1.24 & 1.18 & 1.28 & 10800 \\
\hline & & Intersection Pair 3 & 0.65 & 0.60 & 0.71 & 10800 \\
\hline & & Intersection Pair 4 & 0.88 & 0.83 & 0.93 & 10800 \\
\hline \multirow{4}{*}{ Random } & $\begin{array}{c}\text { Number of Vehicles } \\
\text { to Be Matched }\end{array}$ & NA & 0.81 & 0.15 & 3.34 & 6573 \\
\hline & Traffic Volume & NA & 0.03 & 0.00 & 1.21 & 10800 \\
\hline & $\begin{array}{c}\text { Market } \\
\text { Penetration Rate }\end{array}$ & NA & 0.01 & 0.00 & 0.34 & 10800 \\
\hline & Residual & NA & 0.17 & 0.15 & 0.18 & 10800 \\
\hline
\end{tabular}

